\title{
1 A Performance Evaluation of Nextera XT and KAPA HyperPlus for Rapid 2 Illumina Library Preparation of Long-Range Mitogenome Amplicons
}

\author{
Joseph D. Ring ${ }^{1,2, *}$, Kimberly Sturk-Andreaggi ${ }^{1,2}$, Michelle A. Peck ${ }^{1,2}$, Charla Marshall ${ }^{1,2}$
}

\begin{abstract}
Affiliations:
${ }^{1}$ Armed Forces DNA Identification Laboratory, a division of the Armed Forces Medical Examiner System, 115 Purple Heart Drive, Dover AFB, DE 19902, United States

${ }^{2}$ ARP Sciences, LLC, contractor supporting the Armed Forces Medical Examiner System, 9210 Corporate Boulevard, Suite 120, Rockville, MD 20850, United States

*Corresponding author at: Armed Forces DNA Identification Laboratory, 115 Purple Heart Dr., Dover AFB, DE 19902, United States. Tel.: +1 302346 8506. E-mail address: joseph.d.ring2.ctr@mail.mil
\end{abstract}

\begin{abstract}
$\underline{\text { Abstract }}$
Next-generation sequencing (NGS) facilitates the rapid and high-throughput generation of human mitochondrial genome (mitogenome) data to build population and reference databases for forensic comparisons. To this end, long-range amplification provides an effective method of target enrichment that is amenable to library preparation assays employing DNA fragmentation. This study compared the Nextera XT DNA Library Preparation Kit (Illumina, San Diego, CA) and the KAPA HyperPlus Library Preparation Kit (Kapa Biosystems, Wilmington, MA) for enzymatic fragmentation and indexing of $\sim 8500$ bp mitogenome amplicons for Illumina sequencing. The Nextera XT libraries produced low-coverage regions that were consistent across all samples, while the HyperPlus libraries resulted in uniformly high coverage across the mitogenome, even with reduced-volume reaction conditions. The balanced coverage observed from KAPA HyperPlus libraries enables not only low-level variant calling across the mitogenome but also increased sample multiplexing for greater processing efficiency.
\end{abstract}

\section{Keywords}

Mitochondrial DNA, Next Generation Sequencing (NGS), KAPA HyperPlus, Nextera XT

\section{$\underline{\text { Introduction }}$}

In recent years, next-generation sequencing (NGS) has propelled high-throughput approaches for sequencing the mitochondrial genome (mitogenome). One such method employs long-range (>1000 bp) amplification of mitogenome targets, followed by Nextera XT (Illumina, San Diego, CA) library preparation and Illumina sequencing. Previous studies have shown this method to yield high-quality sequence data that are concordant with mitogenome data generated using "gold-standard" Sanger-type sequencing (STS) approaches [1-4]. However, the Nextera XT assay presents several limitations that minimize sample processing efficiency. First, the tagmentation reaction, which simultaneously fragments and tags DNA using a transposase enzyme, requires $1 \mathrm{ng}$ of DNA input, and therefore individual dilution of each sample's amplicon product is obligatory. Secondly, the Nextera XT procedure relies on limitedcycle PCR for sample indexing [5], introducing the potential for amplification bias [6]. Finally, and perhaps most importantly, Nextera XT mitogenome data show consistent drops in coverage [1-4], 
reflecting a sequence-specific bias of the tagmentation and/or amplification enzyme(s) $[7,8]$. This results in several known regions along the mitogenome with drastically reduced coverage that is independent of haplotype (most notably hypervariable region 2 and np 3562-3592, 5435-5493, 10840-10972, 1303113061, and 13641-13703). In a forensic setting, such uneven coverage can lead to gaps in reportable mitogenome data if the validated minimum coverage threshold is not met. Gaps in coverage may furthermore limit the number of samples that can be multiplexed in a single sequencing run. Therefore depending upon its intended application, Nextera XT may not be an ideal library preparation method for high-throughput mitogenome sequencing.

An alternative library preparation kit from Kapa Biosystems, the HyperPlus Library Preparation Kit (Wilmington, MA), is marketed as a versatile solution for a range of sample types and sequencing applications. The HyperPlus kit utilizes separate enzymatic reactions for DNA fragmentation, end repair, and adapter ligation that enhance its customizability. HyperPlus can accommodate a wide range of DNA input (1-1000 ng), which is preferable when working with forensic samples with varying DNA quantities. Once fragmentation and end-repair of the DNA is complete, adapter ligation can be accomplished using Illumina-compatible, indexed adapters. And unlike Nextera XT, HyperPlus does not require PCR for library preparation, which helps to prevent the introduction of subsequent sequence bias. In a recent study, HyperPlus was shown to produce low fragmentation bias of phiX174 amplicons [9], although its functionality with mitogenome template has not been fully evaluated. This assessment presents a comparison of HyperPlus with Nextera XT using the same long-range amplicons for the purpose of gauging mitogenome sequencing performance.

\section{Material and Methods}

Long-Range Mitogenome Amplification

Ninety-four DNA extracts of anonymized blood serum samples from the Department of Defense Serum Repository were used for this comparison. The use of these samples has been reviewed by the U.S. Army Medical Research and Material Command's Office of Research Protections, Institutional Review Board Office, and was determined not to involve human subjects. Of the 94 samples, 76 were previously sequenced with a Sanger-type mitogenome sequencing procedure [10]. An overlapping, dual-amplicon strategy was employed for long-range amplification of the mitogenome according to the conditions described in Peck et al [4]. One positive control (2800M, Promega, Madison, WI) and one negative control were processed in conjunction with the 94 samples for a total of 96 reactions per amplicon. Amplification product was quantified using the dsDNA $75 \mathrm{bp}-15,000 \mathrm{bp}$ reagent kit (Advanced Analytical, Ames, IA) on a Fragment Analyzer (Advanced Analytical). Both amplicons were combined in equal concentration for each sample. The amplicon pool was then brought to a final working volume of $25 \mu \mathrm{L}$ using $10 \mathrm{mM}$ Tris- $\mathrm{HCl}(\mathrm{pH} 8.5)$ and subsequently purified using a 0.6X AMPure XP (Beckman Coulter, Indianapolis, IN) bead cleanup. The purified amplicon pool was quantified with the Fragment Analyzer dsDNA 75 bp - 15,000 bp reagent kit to assess amplicon concentration prior to library preparation.

\section{Library Preparation}

Nextera XT libraries were prepared in accordance with the manufacturer's protocol [5]. A total of $1 \mathrm{ng}$ DNA input into the Nextera XT reaction was targeted for every sample diluted with $10 \mathrm{mM}$ Tris- $\mathrm{HCl}$ ( $\mathrm{pH} 8.5$ ) in a total volume $5 \mu \mathrm{L}$. HyperPlus libraries were prepared in half-volume reactions with $5 \mu \mathrm{L}$ of undiluted amplicon pool [11]. Half-volume reactions were used to reduce the cost associated with high- 
throughput processing of reference and databasing mitogenome samples (preliminary experiments comparing HyperPlus library preparation at full- and half-volume reactions displayed minimal differences in both fragmentation and sequencing coverage, as shown in Figures S1 and S2). The total fragmentation volume was brought up to $25 \mu \mathrm{L}$ with $10 \mathrm{mM}$ Tris- $\mathrm{HCl}$ (pH 8.5). An incubation of 20 minutes at $37^{\circ} \mathrm{C}$ was used for the fragmentation reaction. Dual-indexed adapters with Illumina TruSeq HT sequences were manufactured by Integrated DNA Technologies (Coralville, IA) and subsequently duplexed in-house. Indexed adapter ligation was performed using an adapter concentration of $15 \mu \mathrm{M}$. After ligation, libraries were purified using a 0.6X AMPure XP bead cleanup to remove unincorporated adapter. No post-library PCR was performed.

\section{Library Quantification, Normalization, and Sequencing}

Libraries were quantified on an Applied Biosystems 7500 Real-Time PCR System (Thermo Fisher Scientific, Waltham, MA) using the KAPA Library Quantification Kit for Illumina Platforms (Kapa Biosystems), with no adjustment for average library fragment size. The fragmentation profiles of the libraries were characterized using the Fragment Analyzer High Sensitivity Large Fragment Analysis Kit (Advanced Analytical). The libraries were individually normalized to $4 \mathrm{nM}$ by diluting each library with $10 \mathrm{mM}$ Tris- $\mathrm{HCl}(\mathrm{pH}$ 8.5) prior to pooling. The HyperPlus and Nextera XT sets were sequenced separately, but both sample pools were prepared and loaded in the same manner. For each set the normalized libraries were pooled by volume, then denatured and diluted to $10 \mathrm{pM}$, and spiked with $10 \mathrm{pM}$ denatured PhiX Sequencing Control V3 (Illumina) at a 5\% concentration (v/v). Each pool was sequenced with a 600-cycle MiSeq Reagent Kit v3 (Illumina) using paired-end sequencing ( 2 x 300 cycles) on a MiSeq FGx Forensic Genomics System (Illumina) in Research Use Only mode.

\section{Data Analysis}

FASTQ files were generated from MiSeq Reporter (Illumina) and the paired reads were imported to the CLC Genomics Workbench v7.5.1 (QIAGEN, Hilden, Germany) for analysis. First, sequences were trimmed $20 \mathrm{bp}$ on both the 3' and 5' ends to remove primers from the read mappings, and sequences shorter than $40 \mathrm{bp}$ were discarded. The trimmed sequences were mapped to the revised Cambridge Reference Sequence (rCRS) [12,13], and a local realignment of unaligned ends was performed to create contiguous indels $[14,15]$. Variants were called with the Low Frequency Variant Detection tool using a $10 \mathrm{X}$ minimum coverage threshold when a minimum variant count of 10 and a minimum variant frequency (VF) of $5 \%$ were satisfied. Consequently, $\geq 200 \mathrm{X}$ coverage was required for $5 \% \mathrm{VF}$ variants to be called. This variant detection strategy was employed to overcome background signal and sequencing error that could result in false heteroplasmies. Additional parameters included ignoring broken pairs, applying a read direction filter of $5 \%$, and applying the default base quality filter (neighborhood radius = 5 , minimum central base quality $=20$, minimum neighborhood base quality $=15$ ). The mitogenome variants were then converted to forensic nomenclature (e.g. 3' shifting of indels and major molecule identification in homopolymeric regions [16]) by a mitochondrial DNA analysis tool custom-designed for the AFDIL by CLC/QIAGEN. For concordance purposes, differences in calling due to length variation, sequencing artifacts resulting from excess primer, and/or minor errors in the alignment of the data were ignored.

130 Target Enrichment and Library Preparation 
A total of 94 samples in addition to a positive and negative control were processed; however one sample was excluded from analysis due to user error during amplicon normalization that resulted in extreme sequencing coverage imbalance between both amplicons. The average pooled amplicon concentration was $47.1 \mathrm{ng} / \mu \mathrm{L} \pm 30.1 \mathrm{ng} / \mu \mathrm{L}$. The sample input for HyperPlus ranged from $11.9 \mathrm{ng}-832.0$ $\mathrm{ng}$ (average of $235.7 \mathrm{ng}$ ), whereas the input for Nextera XT was targeted to be $1 \mathrm{ng}$ per the manufacturer's recommendation (Table 1). This however required more than a 200-fold dilution of the sample on average to reach the Nextera XT target input, whereas no dilution was required before the HyperPlus reaction. Despite the differences in DNA input between HyperPlus and Nextera XT, the resultant library concentrations were similar, and the average DNA library fragment size was larger than 600 bp in both preparations. All samples had a unimodal fragmentation pattern; however HyperPlus produced smaller mode library fragment sizes, as depicted in Figure 1. The small standard deviation of the average mode fragment size in the HyperPlus prepared libraries is noteworthy as well, given the variability in DNA input in the HyperPlus reactions (Figure S3).

Table 1. Library preparation sample metrics for 94 samples presented as the average \pm the standard deviation. The library concentration was determined using the KAPA Library Quantification Kit. All other metrics were measured using the Fragment Analyzer.

\begin{tabular}{|c|c|c|c|c|}
\hline $\begin{array}{c}\text { Library } \\
\text { Preparation }\end{array}$ & $\begin{array}{c}\text { DNA Input } \\
\text { (ng) }\end{array}$ & $\begin{array}{c}\text { Concentration } \\
\text { After Library } \\
\text { Preparation (nM) }\end{array}$ & $\begin{array}{c}\text { Average Fragment } \\
\text { Size After Library } \\
\text { Preparation* (bp) }\end{array}$ & $\begin{array}{c}\text { Mode Fragment Size } \\
\text { After Library } \\
\text { Preparation*(bp) }\end{array}$ \\
\hline HyperPlus & $235.7 \pm 150.4$ & $29.4 \pm 14.1$ & $601 \pm 76$ & $275 \pm 15$ \\
\hline Nextera XT & $\begin{array}{c}1.0 \pm 0.0 \\
\text { (targeted } \\
\text { input) }\end{array}$ & $24.5 \pm 6.4$ & $671 \pm 48$ & $407 \pm 32$ \\
\hline
\end{tabular}

\footnotetext{
$* N=86$ due to limited volume availability in some samples
}

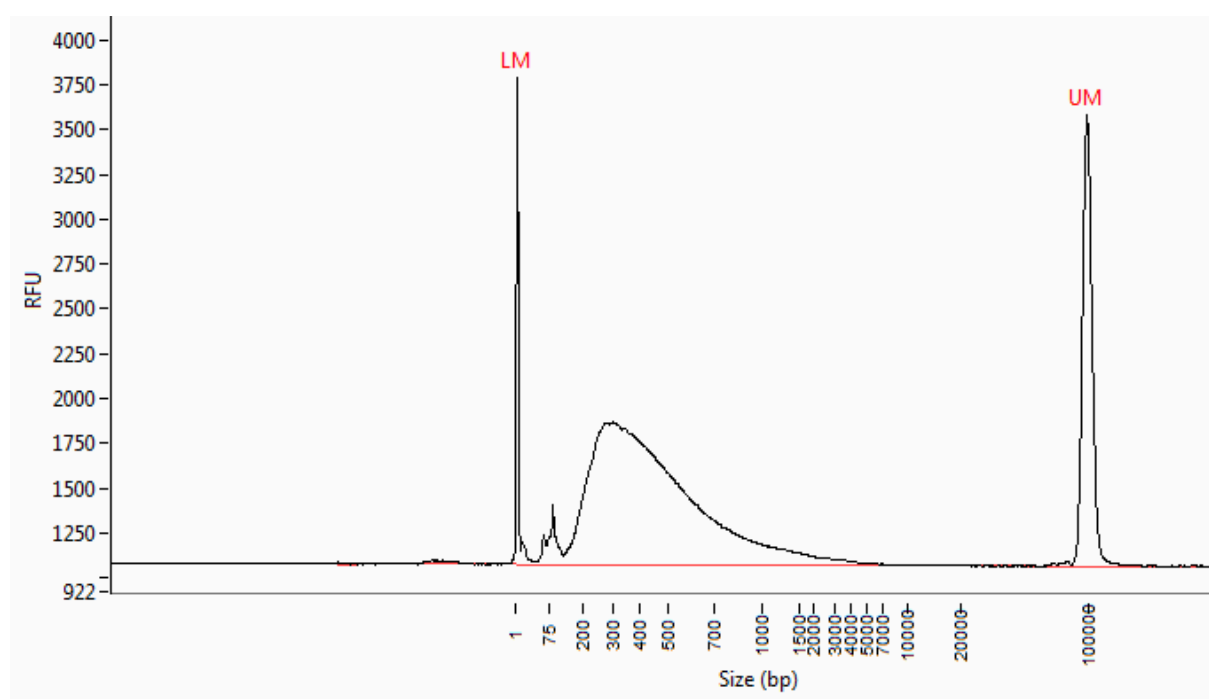




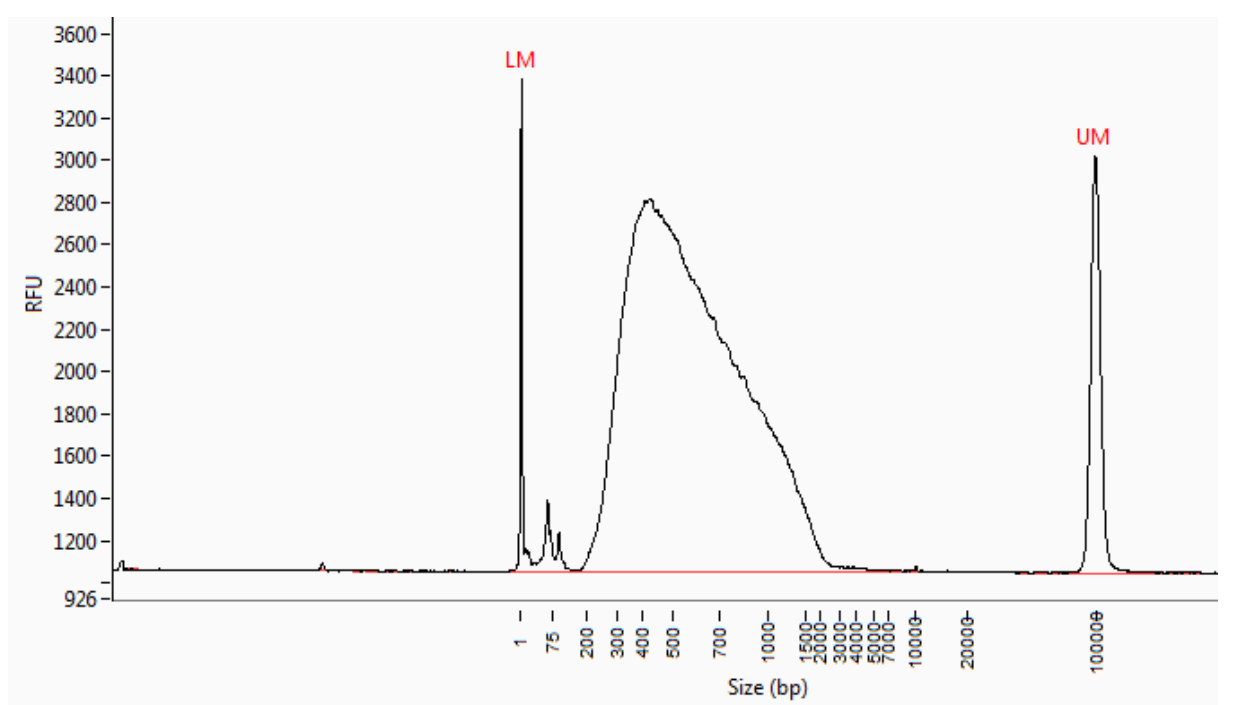

153

154

155

156

157

158

159

160

161

162

163

164

165

166

167

168

169

170

171

172

173

174

175

176

177

178

b.

Figure 1. Representative examples of Fragment Analyzer traces after library preparation with a) HyperPlus and b) Nextera XT. Samples were injected on the Fragment Analyzer using the High Sensitivity Large Fragment Analysis Kit (Advanced Analytical). The Y-axis is the signal strength, measured in relative fluorescence unit (RFU). The lower marker (LM) and upper marker (UM) were injected with each sample as sizing references.

\section{MiSeq Run and Sample Sequencing Metrics}

Both sequencing runs had $>90 \%$ of clusters passing filter (Table 2). However, the cluster density and the correlated number of total sequencing reads for the HyperPlus sequencing run were 1.5-fold higher than the Nextera XT run. The percentage of reads with a Q-score greater than 30 (Q30) was slightly lower in the HyperPlus treatment compared to Nextera XT, although this can be attributed to the shorter HyperPlus libraries having reached sequencing completion prior to the ends of reads 1 and 2 (Figure S4). The difference in these run metrics are likely a result of the differing library composition and/or the accurate molar quantitation of those libraries. Because of this, quantitation of the pooled libraries before loading onto the MiSeq would be beneficial. Regardless, both library preparation methods generated more than 15 million reads and provided ample data for mitogenome analysis.

Table 2. MiSeq run metrics of both library preparation methods.

\begin{tabular}{|c|c|c|c|c|c|}
\hline $\begin{array}{c}\text { Library } \\
\text { Preparation }\end{array}$ & $\begin{array}{c}\text { Cluster Density } \\
\left(\mathbf{k} / \mathbf{m m}^{\mathbf{2}}\right)\end{array}$ & $\begin{array}{c}\text { Clusters Passing } \\
\text { Filter }(\boldsymbol{\%})\end{array}$ & Total Reads & $\begin{array}{c}\text { Reads Passing } \\
\text { Filter }\end{array}$ & \% $>$ Q30 \\
\hline HyperPlus & 1,008 & 93.38 & $24,155,668$ & $22,555,018$ & 63.5 \\
\hline Nextera XT & 655 & 97.23 & $16,100,032$ & $15,652,815$ & 74.8 \\
\hline
\end{tabular}

Table 3 displays the summary sequencing metrics for each method. Each sample garnered roughly $1 \%$ each of the total reads as expected based on equal sample representation in a 96-sample sequencing run. The disparity between HyperPlus and Nextera XT in the percentage of reads retained after end- and quality-trimming is likely due to the difference in sequence length (i.e. more reads trimmed 
in the HyperPlus treatment because of the smaller fragment sizes) or an increased incidence of adapter dimer product. After trimming, an average of over 354,000 reads per sample for HyperPlus and over 291,000 reads per sample for Nextera XT mapped to the rCRS and were used for variant detection.

Table 3. Summary of the sequencing metrics for 94 samples presented as the average \pm the standard deviation.

\section{Coverage Across the Mitogenome}

For both library preparation methods, the average depth of coverage for all samples exceeded 1000X with balanced forward and reverse read coverage (Figure S5). Thus, each kit produced ample sequence data when 96 samples were sequenced simultaneously. Although the average depth of coverage was marginally lower for HyperPlus libraries than the Nextera XT libraries $(1,463 \mathrm{X} \pm 383 \mathrm{X}$ vs. 1,694X \pm $613 \mathrm{X}$, respectively), the variability in coverage across the mitogenome was quite different (Figure 2). As illustrated in Figures 2a-b, the HyperPlus libraries resulted in consistent coverage across the mitogenome, with the exception of the two overlapping regions between the long-range amplicons where the average coverage doubled due to the overlap. Notably, the sequencing coverage did not likewise increase in those same regions of the Nextera XT data, as the transposase enzyme does not add adapters to the distal 50$100 \mathrm{bp}$ of each amplicon during tagmentation [5]. The HyperPlus libraries exceeded 1000X coverage at nearly every nucleotide position except for the section around the polycytosine stretch in the second hypervariable region (np 300-500) where coverage was above 800X. Conversely, the Nextera XT libraries exhibited several regions along the mitogenome with severe drops in coverage, five of which fell below 400X (Figure 2c). In these regions, little standard deviation in coverage across samples was observed, independent of average coverage. The locations of these low coverage regions were consistent with previously published mitogenome data from several laboratories [1-4]. The Nextera XT coverage pattern has been previously attributed to bias in either the Nextera XT transposase reaction [17-20], the library amplification of GC-rich regions [6,18,21,22], or both. Other studies have observed similar coverage imbalance when sequencing alternatively prepared libraries on the Ion Torrent (Thermo Fisher Scientific) sequencing platform [23-25]. Therefore, the combination of HyperPlus library construction and Illumina sequencing facilitates more uniform sequencing coverage of the mitogenome. 


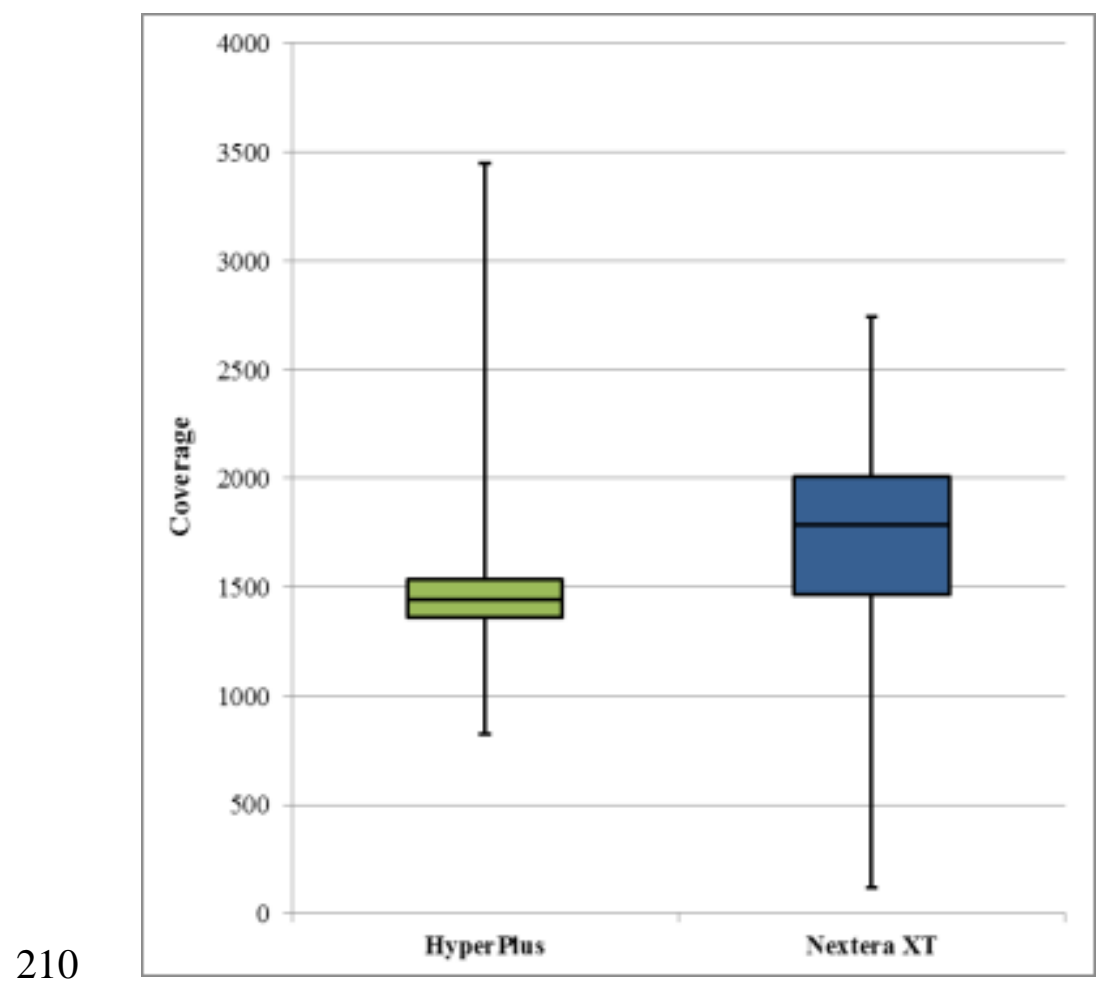

211 a.

212

213

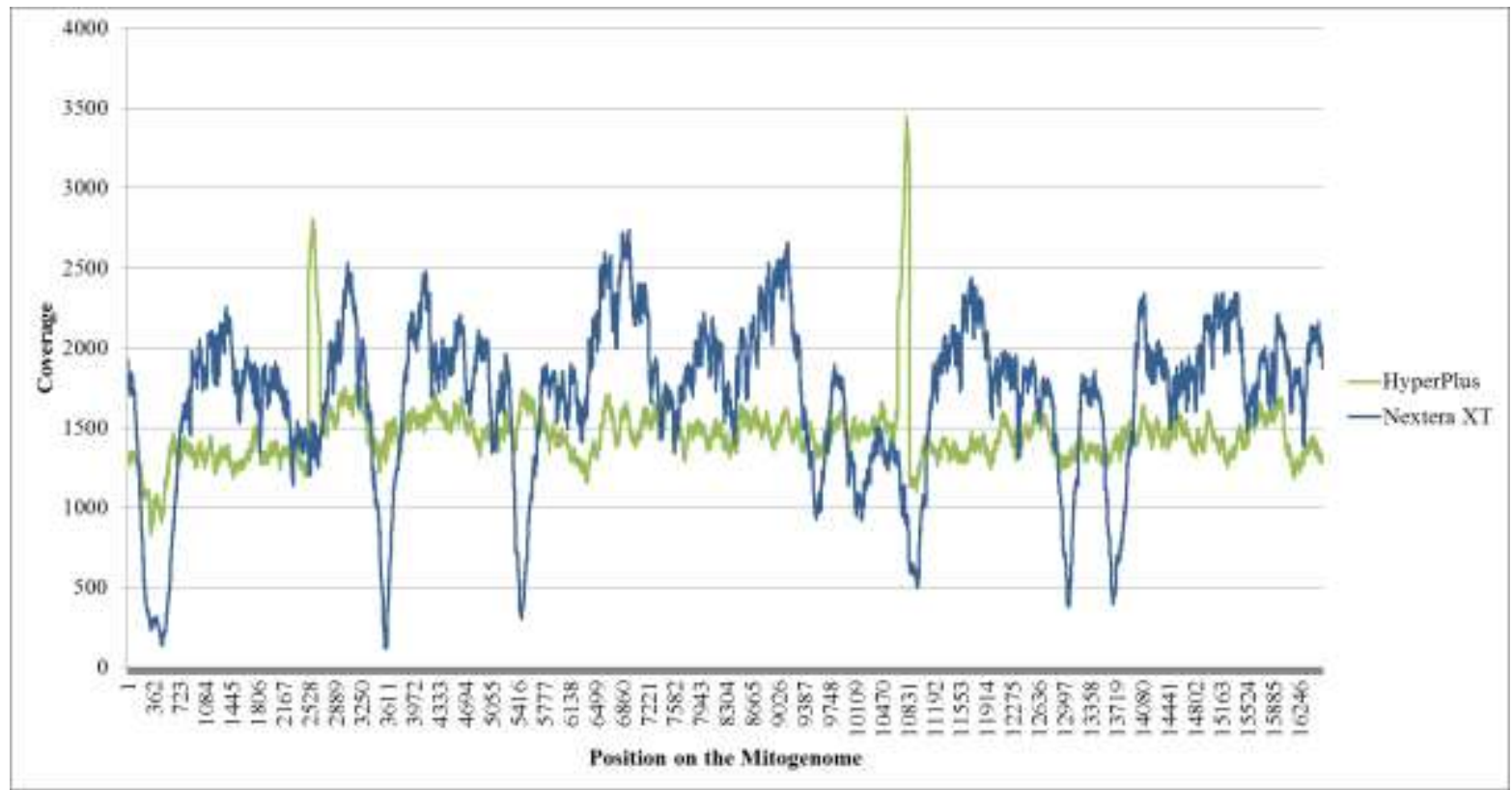

214 b.

215 

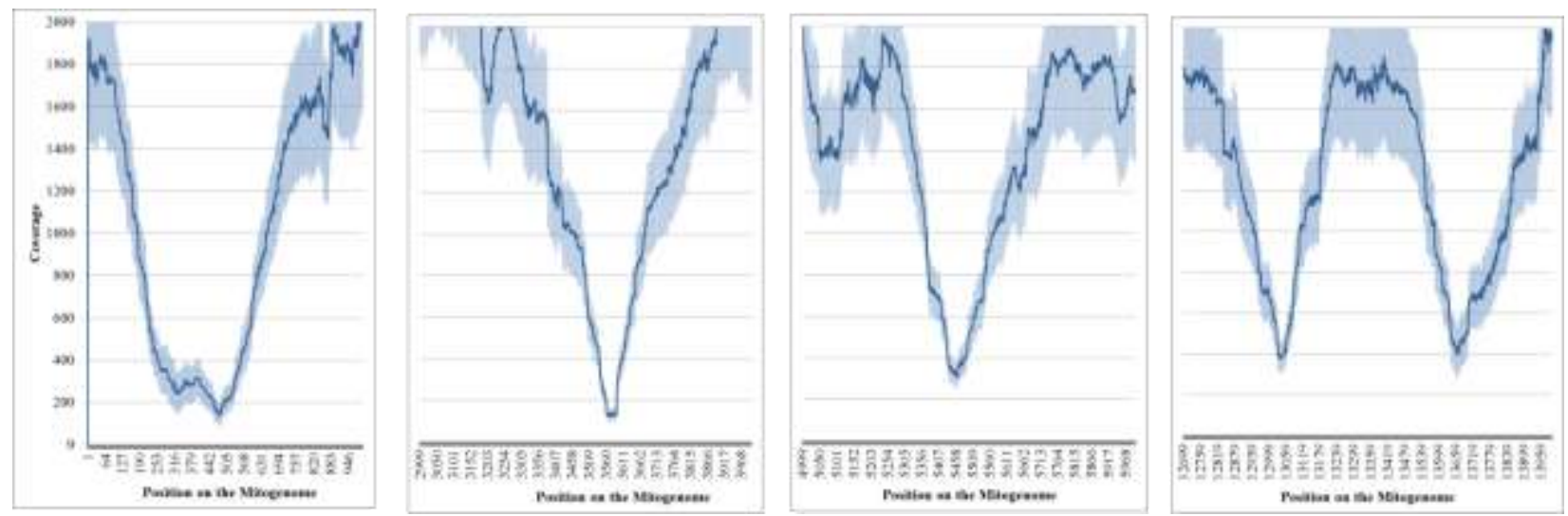

c.

Figure 2. Average coverage analysis for 94 samples across the mitogenome showing a) average coverage box plots, with the error bars depicting the maximum and minimum values in the dataset b) average coverage at each base position across the mitogenome for both Nextera XT and HyperPlus, with the shaded boxes representing the amplicon overlapping regions, and c) average (dark line) and standard deviation (lighter surrounding shade) of the drastically reduced coverage positions for Nextera XT. These figures help exhibit the variable nature of the Nextera XT coverage across the mitogenome (2a-b), yet the inter-sample deviation at the reduced coverage regions was diminutive (2c).

The variability observed in the Nextera XT data led to gaps in low-level variant detection (e.g. $<10 \%)$. The average minimum coverage for Nextera XT-prepared samples was 105X, whereas the average minimum coverage for HyperPlus-prepared samples was approximately seven-fold higher at $784 \mathrm{X}$ (Figure S6). Those positions that failed to meet 200X coverage (a total of 10,212, or 0.66\%, out of all positions across 94 samples) in the Nextera XT data were consequently excluded from low-level variant calling; though higher VF variants meeting the minimum count threshold were still detected.

Depending on the laboratory's application, the detection of low-level variants may be desired. If such a strategy were to be employed, it would increase the minimum coverage threshold requirement at all positions, impacting the Nextera XT data considerably. Table 4 shows a reanalysis of the coverage data using minimum coverage thresholds ranging from $10 \mathrm{X}$ to $1000 \mathrm{X}$. For the HyperPlus samples, 92 of the 94 samples analyzed had complete 500X coverage across the mitogenome. Of the two "incomplete" samples, one had 42 of the 16,569 total positions $(0.25 \%)$ below $500 \mathrm{X}$ and the other sample had a mere four positions below 500X. Five HyperPlus samples had complete 1000X sequencing coverage. Conversely, not a single sample with Nextera XT preparation had complete coverage above 500X, equating to an average of 645 positions (3.89\%) per sample below 500X coverage. Two of the 94 samples treated with Nextera XT had positions below 25X coverage (corresponding to a variant detection limit of only $40 \%$ due to the minimum variant count requirement of 10). Thus, results from these experiments indicate that the HyperPlus library preparation kit produces high minimum coverage across all samples. Extrapolating from the current results, one could theoretically multiplex over 200 samples with KAPA HyperPlus and still maintain a 200X minimum coverage expectation for all nucleotide positions (Table S1). In contrast, Nextera XT sample multiplexing would need to be reduced to no more than 79 samples in order to meet the 200X minimum coverage threshold for 5\% variant detection at all nucleotide positions. 
Table 4. The impact of varying minimum coverage thresholds on mitogenome sequencing.*

\begin{tabular}{|c|c|c|c|c|c|}
\hline \multirow{2}{*}{$\begin{array}{c}\text { Minimum } \\
\text { Coverage } \\
\text { Threshold }\end{array}$} & $\begin{array}{c}\text { VF } \\
\text { Detection } \\
\text { Limit } \uparrow\end{array}$ & $\begin{array}{c}\text { Average Number } \\
\text { of Positions Below } \\
\text { Threshold }\end{array}$ & $\begin{array}{c}\text { Samples with } \\
\text { Complete } \\
\text { Coverage of All } \\
\text { Positions }\end{array}$ & $\begin{array}{c}\text { Hverage Number } \\
\text { of Positions Below } \\
\text { Threshold }\end{array}$ & $\begin{array}{c}\text { Samples with } \\
\text { Complete } \\
\text { Coverage of All } \\
\text { Positions }\end{array}$ \\
\hline $1000 \mathrm{X}$ & $1 \%$ & $1311 \pm 2761$ & $5 / 94$ & $2009 \pm 666$ & $0 / 94$ \\
\hline $500 \mathrm{X}$ & $2 \%$ & $0 \pm 4$ & $92 / 94$ & $645 \pm 157$ & $0 / 94$ \\
\hline $200 \mathrm{X}$ & $5 \%$ & $0 \pm 0$ & $94 / 94$ & $364 \pm 113$ & $1 / 94$ \\
\hline $100 \mathrm{X}$ & $10 \%$ & $0 \pm 0$ & $94 / 94$ & $10 \pm 21$ & $54 / 94$ \\
\hline $50 \mathrm{X}$ & $20 \%$ & $0 \pm 0$ & $94 / 94$ & $1 \pm 9$ & $91 / 94$ \\
\hline $25 \mathrm{X}$ & $40 \%$ & $0 \pm 0$ & $94 / 94$ & $0 \pm 3$ & $92 / 94$ \\
\hline $10 \mathrm{X}$ & $100 \%$ & $0 \pm 0$ & $94 / 94$ & $0 \pm 0$ & $94 / 94$ \\
\hline
\end{tabular}

* Row shaded gray is the analysis threshold utilized in this study

$\dagger$ With a minimum variant count of 10

\section{Concordance}

All samples and the positive control were concordant between Nextera XT and KAPA HyperPlus, with only minimal fluctuation in the VF of any low-level variants (Table S2). Of the 75 samples with STS data available for comparison, two had low-level control region (CR) point heteroplasmies (PHPs) called in the STS data that were not identified in one or both sets of the NGS data (yet still present at a VF less than 5\%). In Peck et al. which followed the same amplification strategy, each instance of PHP that was called in the STS data but not in the NGS data was at a position located in the CR [4]. It may be that differences in amplification conditions between the two methods (e.g. polymerase, thermal cycling parameters, amplicon size) contributed to differences in sensitivity and/or induced more stochastic variation in that region. The remaining STS variants were reproduced in both the HyperPlus and Nextera XT mitogenome data. The positive control had full coverage for both conditions, and the variants were concordant with those expected. However, the negative control was shown in both library preparations to be contaminated with one of the samples directly next to it on the long-range amplification plate. The average coverage was $35 \mathrm{X}$ in the Nextera XT negative control and $3 \mathrm{X}$ in the HyperPlus control, well below the average of any sample. The contamination event likely occurred prior to the library preparation reactions, and no additional evidence of cross-contamination was observed in any other sample. The contaminated control highlights the susceptibility for cross-contamination in manual, high-throughput NGS sample preparation.

\section{Conclusions}

Utilizing NGS for mitogenome sequencing enables increased throughput and data generation compared to STS methods. Kapa Biosystems' KAPA HyperPlus and Illumina's Nextera XT kits are similarly priced and are both easily amenable to automation. However when moving toward the adoption of NGS for forensic casework, a balance must be struck between throughput and coverage. When tested on the same long-range amplicons, HyperPlus resulted in consistent, high coverage of the mitogenome due to its sequence-agnostic chemistry, even at half-volume reactions. Conversely, Nextera XT resulted in several low-coverage regions along the mitogenome. The consistent coverage of HyperPlus allowed for $5 \%$ VF variant detection across the mitogenome, whereas the drops in coverage observed in the Nextera 
XT data limited low-level variant calling. HyperPlus furthermore provides a more streamlined and efficient laboratory workflow that accommodates a wide DNA input range, eliminates the need for a postlibrary PCR step, and allows for increased sample multiplexing. These advantages of HyperPlus may therefore translate to per-sample cost savings, which could be further enhanced with high-throughput automation. In sum, the mitogenome sequencing performance displayed by HyperPlus libraries on the Illumina MiSeq demonstrates the potential for gains in sequencing efficiency without compromising data quality or consistency in coverage that enables uniform variant detection.

\section{Acknowledgments}

The authors would like to thank Erin Gorden and Jennifer Higginbotham (ARP Sciences, LLC, Armed Forces DNA Identification Laboratory) for laboratory and analysis assistance; Timmathy Cambridge (Armed Forces Medical Examiner System) for technical assistance; Michael Fasano, James Canik, and Cynthia Thomas (ARP Sciences, LLC), Timothy McMahon, Shairose Lalani, Lt Col Garner, Lt Col Briones, COL Ladd Tremaine, and COL Louis Finelli (Armed Forces Medical Examiner System) for administrative and logistical support, and Ken Kroupa and the Rapid Reaction Technology Office, ASD (R\&E) for funding. We also would like to thank the two anonymous reviewers whose expertise, comments, and suggestions for revision improved this paper. The opinions or assertions presented hereafter are the private views of the authors and should not be construed as official or as reflecting the views of the Department of Defense, its branches, the U.S. Army Medical Research and Materiel Command, the Defense Health Agency, or the Armed Forces Medical Examiner System.

\section{$\underline{\text { References }}$}

[1] K. Vancampenhout, B. Caljon, C. Spits, K. Stouffs, A. Jonckheere, L. De Meirleir, et al., A bumpy ride on the diagnostic bench of massive parallel sequencing, the case of the mitochondrial genome, PLoS One. 9 (2014) e112950.

[2] J.L. King, B.L. LaRue, N.M. Novroski, M. Stoljarova, S.B. Seo, X. Zeng, et al., High-quality and high-throughput massively parallel sequencing of the human mitochondrial genome using the Illumina MiSeq, Forensic.Sci.Int.Genet. 12 (2014) 128-135.

[3] J.A. McElhoe, M.M. Holland, K.D. Makova, M.S. Su, I.M. Paul, C.H. Baker, et al., Development and assessment of an optimized next-generation DNA sequencing approach for the mtgenome using the Illumina MiSeq, Forensic.Sci.Int.Genet. 13 (2014) 20-29.

[4] M.A. Peck, M.D. Brandhagen, C. Marshall, T.M. Diegoli, J.A. Irwin, K. Sturk-Andreaggi, Concordance and reproducibility of a next generation mtGenome sequencing method for high-quality samples using the Illumina MiSeq, Forensic.Sci.Int.Genet. 24 (2016) 103-111.

[5] Illumina, Nextera XT DNA Library Preparation Guide, Rev. E ed., Illumina 2015. Illumina sequencing-library preparation facilitates improved mapping and assembly of $(\mathrm{G}+\mathrm{C})$-biased genomes, Nat.Methods. 6 (2009) 291-295. 
322

323

324

325

326

327

328

329

330

331

332

333

334

335

336

337

338

339

340

341

342

343

344

345

346

[8] R. Marine, S.W. Polson, J. Ravel, G. Hatfull, D. Russell, M. Sullivan, et al., Evaluation of a transposase protocol for rapid generation of shotgun high-throughput sequencing libraries from nanogram quantities of DNA, Appl.Environ.Microbiol. 77 (2011) 8071-8079.

[9] L. Aigrain, Y. Gu, M.A. Quail, Quantitation of next generation sequencing library preparation protocol efficiencies using droplet digital PCR assays - a systematic comparison of DNA library preparation kits for Illumina sequencing, BMC Genomics. 17 (2016) 458-016-2757-4.

[10] R.S. Just, M.K. Scheible, S.A. Fast, K. Sturk-Andreaggi, A.W. Rock, J.M. Bush, et al., Full mtGenome reference data: development and characterization of 588 forensic-quality haplotypes representing three U.S. populations, Forensic.Sci.Int.Genet. 14 (2015) 141-155.

[11] Kapa Biosystems, KAPA HyperPlus Library Preparation Kit Technical Data Sheet, v1.15 ed., Kapa Biosystems 2015.

[12] S. Anderson, A.T. Bankier, B.G. Barrell, M.H. de Bruijn, A.R. Coulson, J. Drouin, et al., Sequence and organization of the human mitochondrial genome, Nature. 290 (1981) 457-465.

[13] R.M. Andrews, I. Kubacka, P.F. Chinnery, R.N. Lightowlers, D.M. Turnbull, N. Howell, Reanalysis and revision of the Cambridge reference sequence for human mitochondrial DNA, Nat.Genet. 23 (1999) 147.

[14] QIAGEN, CLC Genomics Workbench User Manual, Version 7.5.1 ed. 2014.

[15] N. Homer, S.F. Nelson, Improved variant discovery through local re-alignment of short-read nextgeneration sequencing data using SRMA, Genome Biol. 11 (2010) R99-2010-11-10-r99. Epub 2010 Oct 8 .

[16] W. Parson, L. Gusmao, D.R. Hares, J.A. Irwin, W.R. Mayr, N. Morling, et al., DNA Commission of the International Society for Forensic Genetics: revised and extended guidelines for mitochondrial DNA typing, Forensic.Sci.Int.Genet. 13 (2014) 134-142.

[17] I.Y. Goryshin, J.A. Miller, Y.V. Kil, V.A. Lanzov, W.S. Reznikoff, Tn5/IS50 target recognition, Proc.Natl.Acad.Sci.U.S.A. 95 (1998) 10716-10721.

[18] A. Adey, H.G. Morrison, Asan, X. Xun, J.O. Kitzman, E.H. Turner, et al., Rapid, low-input, low-bias construction of shotgun fragment libraries by high-density in vitro transposition, Genome Biol. 11 (2010) R119-2010-11-12-r119. Epub 2010 Dec 8.

[19] S. Lamble, E. Batty, M. Attar, D. Buck, R. Bowden, G. Lunter, et al., Improved workflows for high throughput library preparation using the transposome-based Nextera system, BMC Biotechnol. 13 (2013) 104-6750-13-104.

[20] A.D. Tyler, S. Christianson, N.C. Knox, P. Mabon, J. Wolfe, G. Van Domselaar, et al., Comparison of Sample Preparation Methods Used for the Next-Generation Sequencing of Mycobacterium tuberculosis, PLoS One. 11 (2016) e0148676.

[21] M.A. Quail, T.D. Otto, Y. Gu, S.R. Harris, T.F. Skelly, J.A. McQuillan, et al., Optimal enzymes for amplifying sequencing libraries, Nat.Methods. 9 (2011) 10-11. 
358 [22] S.O. Oyola, T.D. Otto, Y. Gu, G. Maslen, M. Manske, S. Campino, et al., Optimizing Illumina next359 generation sequencing library preparation for extremely AT-biased genomes, BMC Genomics. 13 (2012)

$360 \quad 1-2164-13-1$.

361 [23] S.B. Seo, X. Zeng, J.L. King, B.L. Larue, M. Assidi, M.H. Al-Qahtani, et al., Underlying Data for 362 Sequencing the Mitochondrial Genome with the Massively Parallel Sequencing Platform Ion Torrent 363 PGM, BMC Genomics. 16 Suppl 1 (2015) S4-2164-16-S1-S4. Epub 2015 Jan 15.

364 [24] Y. Zhou, F. Guo, J. Yu, F. Liu, B. Zhao, F. Jia, et al., Strategies for complete mitochondrial genome 365 sequencing on Ion Torrent PGM platform in forensic sciences. Forensic.Sci.Int.Genet. 22 (2016) 11-21.

366 [25] J.D. Churchill, J.L. King, R. Chakraborty, B. Budowle, Effects of the Ion PGM Hi-Q sequencing 367 chemistry on sequence data quality. Int. J. Legal Med. 130 (2016) 1169-1180. 